\title{
Desynchronized stable states in diluted neural networks
}

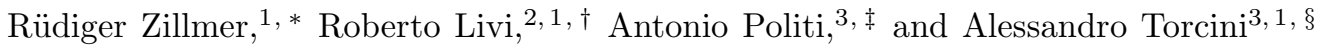 \\ ${ }^{1}$ INFN Sez. Firenze, via Sansone, 1 - I-50019 Sesto Fiorentino, Italy \\ ${ }^{2}$ Dipartimento di Fisica - CSDC, Universitá di Firenze, \\ via Sansone, 1 - I-50019 Sesto Fiorentino, Italy \\ ${ }^{3}$ Istituto dei Sistemi Complessi, CNR, via Madonna del Piano 10, I-50019 Sesto Fiorentino, Italy
}

\begin{abstract}
The dynamical properties of a diluted fully-inhibitory network of pulse-coupled neurons are investigated. Depending on the coupling strength, two different phases can be observed. At low coupling the evolution rapidly converges towards periodic attractors where all neurons fire with the same rate. At larger couplings, a new phase emerges, where all neurons are mutually unlocked. The irregular behaviour turns out to be "confined" to an exponentially long, stationary and linearly stable transient. In this latter phase we also find an exponentially tailed distribution of the interspike intervals (ISIs). Finally, we show that in the unlocked phase a subset of the neurons can be eventually synchronized under the action of an external signal, the remaining part of the neurons acting as a background noise. The dynamics of these "background" neurons is quite peculiar, in that it reveals a broad ISI distribution with a coefficient of variation that is close to 1 .
\end{abstract}

Keywords: Puse-coupled inhibitory network, Random dilution, Multistability, Long chaotic transients

\section{INTRODUCTION}

A challenging task in the context of complex neural networks is the comprehension of how their dynamical features are influenced by their structure. A good strategy to tackle the problem consists in studying simple models, since one can more easily identify the minimal and relevant ingredients that are responsible for generic properties. In this work we investigate a diluted neural network of pulse-coupled neurons. ${ }^{1}$ Since inhibition plays an important role in the dynamics of neurons in vivo 3, 4], we have chosen to examine a network of inhibitory coupled leaky integrate-and-fire neurons. Recently, it has been shown that dilution can induce long chaotic transients in excitatory networks with delay [13]. We aim at understanding the new features emerging in the dynamics of a network where two competitive effects like dilution and full inhibition coexist. We show that the maximum Lyapunov exponent is negative ${ }^{2}$, so that the evolution eventually converges onto a periodic attractor. This notwithstanding, the dynamics is far from trivial, because above a given threshold, the transient grows exponentially with the system size and is characterized by seemingly statonary properties. These features altogether, plus the stochastic-like nature of the transient, suggest a strong analogy with stable chaos (SC), a phe-

\footnotetext{
*Electronic address: zillmer@fi.infn.it

${ }^{\dagger}$ Electronic address: livi@fi.infn.it

${ }^{\ddagger}$ Electronic address: antonio.politi@isc.cnr.it

$\S$ Electronic address: alessandro.torcini@isc.cnr.it

${ }^{1}$ Here dilution refers to a random pruning of directed links in a globally coupled network.

2 Strictly speaking, it is the second exponent, as there exists an exponent equal to zero that corresponds to the motion along the trajectory. For the sake of clarity and since we study the dynamics emerging from the introduction of a Poincaré section, we always disregard the zero exponent.
}

nomenon discovered in the context of coupled map lattices [8]. The possibility of observing SC in the neuroscience context is important because this regime is both stable to external perturbations and characterized by a richness of behaviour depending on the initial conditons: both properties are welcome for a system that is expected to reliably perform universal computations. The present case study can be considered as the first example of SC in an autonomous continuous-time system. In fact, it was formerly believed that SC can arise only in periodically forced systems, as one must simultaneously have a zero Lyapunov exponent (in order to ensure a meaningful dynamics different from a dull fixed point) and a strictly negative second Lyapunov exponent. The continuity of Lyapunov spectra in autonomous spatially extended systems prevents the above condition to be fulfilled and, in fact, the only previous SC example in a continuous time system is a chain of periodically forced Duffing oscillators [1]. However, these arguments do not apply to globally coupled systems and this explains why SC can and has been observed in neural networks.

\section{DYNAMICS OF A WEAKLY DISORDERED NETWORK}

We consider a system made of $N$ leaky integrate-andfire neurons, which interact by sending each other pulses through a directed graph of connections. The non-zero elements of the connectivity matrix are $G_{i j}=G_{0} / \ell_{i}$, where $G_{0}$ is the coupling constant and $\ell_{i}$ is the number of incoming links to neuron $i$. Such a normalization condition has been used in the literature when dealing with randomly connected networks [5, 13]. The membrane potential $v_{i}(t)$ of the $i$-th neuron obeys the dynamical rule 


$$
\dot{v}_{i}=c-v_{i}-\left(v_{i}+w\right) \sum_{j=1}^{N} \sum_{m=1}^{M_{j}} G_{i j} \delta\left(t-t_{j}^{(m)}\right) ;
$$

where we use dimensionless quantities (details about the derivation of the model can be found in 12]). The suprathreshold input current, $c$, and the reversal potential, $w$, are assumed to take the values 2 and 4/7, respectively. This choice was derived from values used in the current literature (see e.g., [2, 6]). The potential $v_{i}(t)$ follows the above dynamics until it reaches the threshold value 1 , whereupon the neuron emits a spike and the potential is reset to the value 0 . The integer $M_{j}$ in the coupling term counts the spikes emitted by neuron $j$ at the time $t_{j}^{(m)}$. Since we assume $G_{0}>0$ and $w>0$, the coupling turns out to be fully inhibitory.

One further important ingredient of the model is disorder that is introduced by randomly pruning a fraction $r_{\mathrm{p}}$ of directed links. As a matter of fact, the main control parameters are $r_{\mathrm{p}}$ and the coupling constant $G_{0}$.

By introducing a Poincaré section (defined as the manifold where a generic neuron potential reaches the threshold), it has been shown that the network dynamics can be reduced to a discrete map of the potentials $v_{i}(n)$ between successive spikes 12. Accordingly, one can replace the continuous time axis with an integer $n$ denoting the $n$th spike. It is convenient to simulate the model by directly iterating the resulting map.

A preliminary analysis has been performed in Ref. [6], where it was shown that the evolution converges to a periodic state and rigorous upper bounds for the transient time have been thereupon derived. Accordingly, the dynamics seems to be quite straightforward and in fact the maximum Lyapunov exponent remains negative even in the thermodynamic limit [12]. More precisely, a homogeneous fully coupled network rapidly converges towards a periodic phase with all neurons firing with the same rate. On the other hand, the transient length may become exponentially long with the system size, so that in large networks the name transient becomes inadequate to identify what turns out to be a long-lasting stationary regime. Interestingly enough, upon increasing the amount of disorder (i.e., by increasing the fraction $r_{\mathrm{p}}$ of cut links) one passes from the former to the latter regime. The results reported in this section for $r_{\mathrm{p}}$ as small as 0.05 , show that for sufficiently large coupling strengths a stochastic-like behaviour is self-generated.

At low coupling strengths (up to $G_{0} \lesssim 1$ ), the evolution is substantially equivalent to that of a fully coupled system. After a short transient time, on the order of the system size $N$, all neurons fire with the same pace $\left(t_{\text {isi }}=\right.$ const $)$ but are characterized by different absolute phases. The main difference between this synchronized regime, that we call Locked Phase (LP), and that one occurring in the fully coupled case is the difference among the relative phases. In fact, the equivalence among all neurons of a homegeneous network forces the evolution to converge towards a highly symmetric state characterized not only by a constant interspike interval (ISI) $t_{\text {isi }}$, but also by a constant time separation between consecutive spikes. On the other hand, already the introduction of a small amount of disorder breaks the symmetry, and different spiking sequences are no longer equivalent to one another, with the consequence that slightly different time series are generated for different initial conditions. Although this phase is dynamically trivial, it may have some relevance from the point of view of information storage, since there is an exponentially large (and even more than that) number of different attractors that may be selected by means of suitable initial inputs.

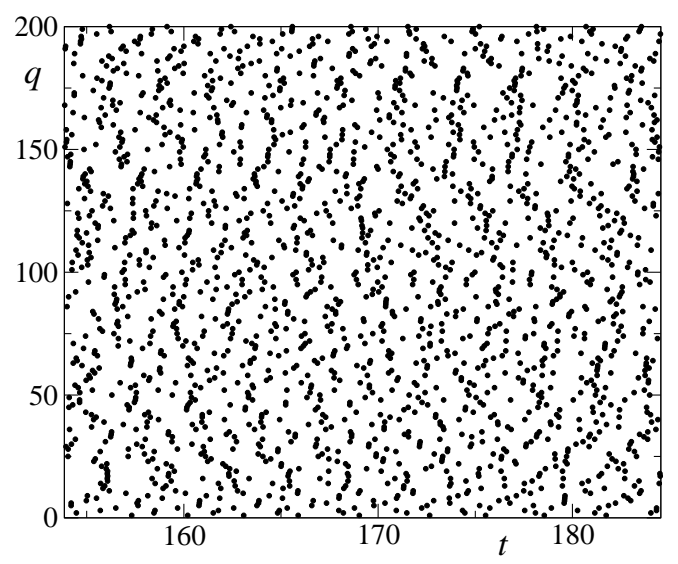

FIG. 1: Firing pattern during the transient in the UP $\left(G_{0}=\right.$ $\left.2, N=200, r_{\mathrm{p}}=0.05\right) . q$ denotes the index of the firing neuron.

At higher coupling strengths $\left(G_{0} \gtrsim 1\right)$ a different network dynamics sets in. The ISI is no-longer constant and the resulting pattern keeps slowly changing (e.g., see Fig. 11). We accordingly call this dynamical regime Unlocked Phase (UP) . The phase variables corresponding to the different neurons evolve like independent random walks, despite the dynamics is characterized by a negative maximum Lyapunov exponent. One can understand the new scenario by observing that the increased (inhibitory) coupling strength destabilizes (or just makes disappear) an increasing number of periodic spiking sequences. Accordingly, we observe a continuous rearrangement of the ordering, until one of the remaining stable sequences is reached. It is certainly desirable to understand the rules of this pruning of periodic solutions, but we first prefer to concentrate our efforts on the statistical characterization of the transient regime, since the irregularity of the evolution can neither be traced back to an exponential separation of orbits, nor to the action of an external noise source. We start by computing the normalized autocorrelation function of the ISIs, $t_{\mathrm{isi}}(n)$, of the single neurons,

$$
C_{\mathrm{isi}}(n)=\frac{\left\langle t_{\mathrm{isi}}\left(n^{\prime}\right) t_{\mathrm{isi}}\left(n^{\prime}+n\right)\right\rangle-\left\langle t_{\mathrm{isi}}\right\rangle^{2}}{\left\langle t_{\mathrm{isi}}^{2}\right\rangle-\left\langle t_{\mathrm{isi}}\right\rangle^{2}} ;
$$


where $\langle\cdot\rangle$ denotes the average over the time index $n^{\prime}$. $C_{\text {isi }}$ (see Fig. 2) clearly exhibits an exponential decay, while the corresponding ISI distribution (see the inset in the same figure) exhibits an exponential tail that is typical of Poisson processes and testifies to the absence of correlations at long times.

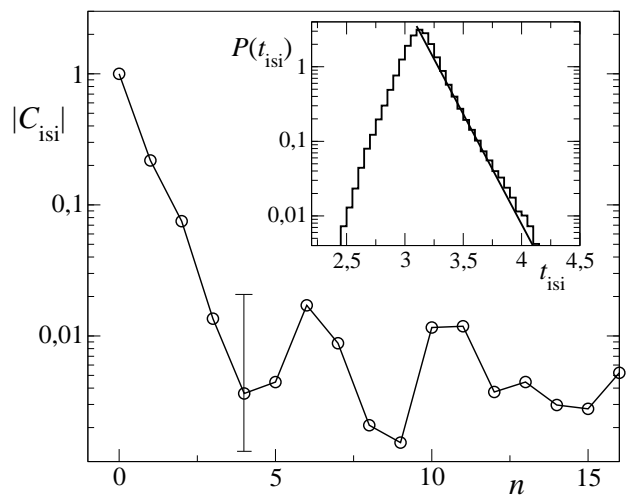

FIG. 2: The normalized autocorrelation $C_{\text {isi }}$ of the $t_{\text {isi }}$ during transient for a single neuron vs. the integer delay for $N=$ $1000, G_{0}=2$. The inset shows the corresponding distribution $P\left(t_{\text {isi }}\right)$ that exhibits an exponential decay.

It is also important to test the stationarity of the UP. By computing the coefficient of variation, $C_{\mathrm{V}}(n)$ (the standard deviation normalized to the mean value) of the ISI in different windows, it can be seen that while $C_{\mathrm{V}}(n)$ slowly decreases towards 0 in the LP (indicating a convergence towards an ordered state), it remains constant in the UP [12] although the asymptotic value is quite small. For instance, we find that $C_{\mathrm{V}} \approx 0.03$ for $G_{0}=1.6$ in contrast to values around 1, typically found for randomly spiking neurons in the cortex [9]. This can be due to the smallness of the disorder that is considered in the network. However, it is also interesting to notice that even in the present context for a different setup (i.e., under the action of a periodic input) a much wider distribution can be found as discussed in the next section.

\section{RESPONSE TO A PERIODIC SIGNAL}

An interesting question is whether the emergence of long irregular transients in the presence of multiple periodic attractors allows performing neurocomputational tasks. As a first test in this direction we study the effect of a periodic signal on a subset $N^{\prime}$ of the $N$ neurons. The signal is assumed to be an equispaced spike train with an excitatory action on the receiving neurons. The dynamics of the unforced $N-N^{\prime}$ neurons is still given by Eq. (1), while the remaining $N^{\prime}$ neurons are subject to the extra excitatory synaptic input,

$$
I_{e}=-\left(v_{i^{\prime}}+w_{\mathrm{e}}\right) G_{\mathrm{e}} \sum_{n=1}^{M_{\mathrm{e}}} \delta\left(t-t_{\mathrm{e}}^{(n)}\right)
$$

where $w_{\mathrm{e}}$ is the reversal potential, $G_{\mathrm{e}}$ represents the coupling constant and $M_{\mathrm{e}}$ counts the external spikes received up to the time $t$. The ISI of the excitatory post-synaptic potentials (PSPs) is assumed to be constant (i.e., $t_{\mathrm{e}}^{(n+1)}-t_{\mathrm{e}}^{(n)} \equiv T_{\mathrm{e}}$ ). Thus the effective signal strength is given by the ratio $G_{\mathrm{e}} / T_{\mathrm{e}}$.

Due to the excitatory nature of the PSP, the firing rate of the $N^{\prime}$ neurons increases, and this, in turn, gives rise to an increased inhibitory activity in the network. Thus the activity of the $N-N^{\prime}$ "background" neurons that do not partake in the excitatory input should diminish compared to the subset $N^{\prime}$. In Fig. 3 we present results for a system of $N=500$ neurons in the UP $\left(G_{0}=1.8\right)$. The input signal is switched on during the time interval $t \in[25.9,31.8]$; otherwise the network evolves freely according to Eq. (11). For the PSP input trains, we have taken the values $w_{\mathrm{e}}=-8, T_{\mathrm{e}}=10^{-3}$ and $G_{\mathrm{e}}=2.2 \times 10^{-4}$, i.e., $G_{\mathrm{e}} / T_{\mathrm{e}}=0.22$. The input is applied to the first 50 neurons (given the equivalence on average among all neurons, the choice of the label is totally irrelevant); accordingly $N^{\prime} / N=0.1$. By looking at the firing pattern shown in Fig. B3(a), one can clearly notice the effect of the excitatory input from the significantly increased firing frequency of the driven neurons, while, simultaneously, the activity of the undriven background neurons diminishes. Not surprisingly, the pattern of the $N^{\prime}$ neurons is much more regular than the spike pattern of the background. This is better seen by looking at the ISI's, $t_{\text {isi }}^{\prime}$, of the driven neurons in Fig. 3(b). Indeed, soon after the input has been switched on the $t_{\text {isi }}^{\prime}$ rapidly decrease towards 0.43 with small fluctuations $<2 \%$. Once the signal has been switched off, the system rapidly returns to the initial irregular pattern after the neurons have been reset.

When the input signal is on, the forced neurons behave almost periodically with a period $t_{\text {isi }}^{\prime} \gg T_{\mathrm{e}}$. The small fluctuations are due to the inhibitory action of the background neurons. However, the negative Lyapunov exponent ensures stability with respect to small perturbations and thereby guarantees a robust synchronization of the subset. The $N^{\prime}$ neurons somehow resemble the "active" population of cells that respond to a certain stimulus by firing with an increased rate.

On the other hand, the ISIs of the background are more irregular than in the freely evolving network as revealed by the strongly enhanced fluctuations. In order to perform a more accurate description of their behavior, we have switched on the input for longer times, so as to be able to determine the probability distribution of the intervals $t_{\text {isi }}$ in the stationary regime. It turns out that different background neurons show substantial differences in their firing rate, $\left\langle t_{\text {isi }}\right\rangle^{-1}$. In Fig. 过(a) the distribution of $\left\langle t_{\text {isi }}\right\rangle$ is shown, that can be fitted for intermediate values by a power-law decay with an exponent $\approx 3$. However, the tail shows significant deviations from the power-law, which suggests a decomposition of the background neurons into different classes. In order to get a deeper insigth into this phenomenon, we have examined the distribution 
(a)

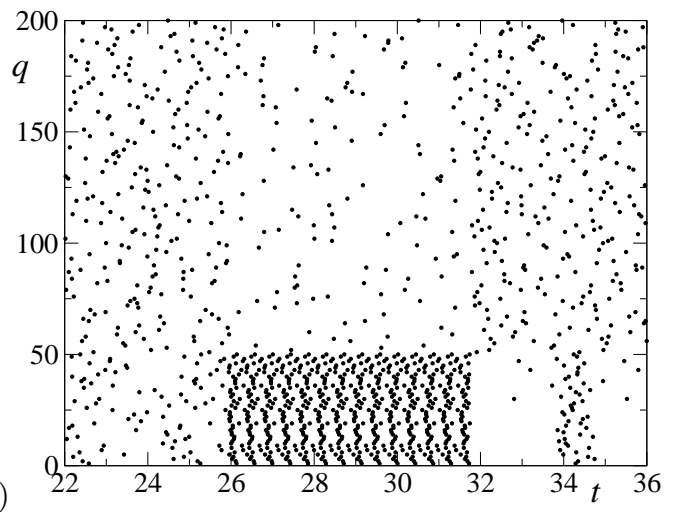

(b)

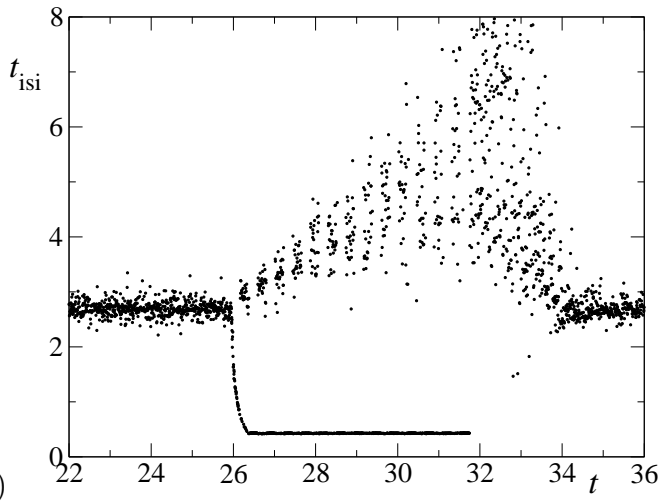

FIG. 3: (a) Firing pattern for $G_{0}=1.8, N=500 ; q$ denotes the index of the firing neuron. The excitatory input was appplied to neurons $i=1, \cdots 50$ for $t \in[25.9,31.8]$. To enhance visibility only the first 200 neurons are shown. (b) The $t_{\text {isi }}$ of the respective firing neuron. With the applied signal the $t_{\text {isi }}$ of the receiving neurons lock to $t_{\mathrm{isi}}^{\prime}=0.43$.

of single neuron $C_{\mathrm{V}}$ 's for different system sizes keeping $N^{\prime} / N=0.1$ (see Fig. प(b)). As a matter of fact, this characterization of the firing processes reveals a bimodal distribution. The peak seen at smaller values is reminiscent of the behaviour of the freely evolving network: we can claim that such neurons are almost unaffected by the input signal. On the other hand, the neurons with $C_{\mathrm{V}} \simeq 1$ exhibit a Poissonian ISI distribution with a much more pronounced exponential tail. The different behavior of background neurons can be understood by examining for each neuron the number of intact incoming links from forced neurons, that after a normalization with $N^{\prime}$ yields the local connectivity $r_{\text {con }} \in[0,1]$. A large value of $r_{\text {con }}$ implies an enhanced inhibition of the respective neuron. We collected neurons that share the same value of $r_{\text {con }}$ and computed the corresponding average $C_{\mathrm{V}}$ that we denote as $\left\langle C_{\mathrm{V}}\right\rangle_{\mathrm{s}}$. Indeed, the results in Fig. प4(c) show that with increasing $r_{\text {con }}$ there is a transition-like behavior of $\left\langle C_{\mathrm{V}}\right\rangle_{\mathrm{s}}$, as it rapidly increases from values around 0.05 to values around 1 .

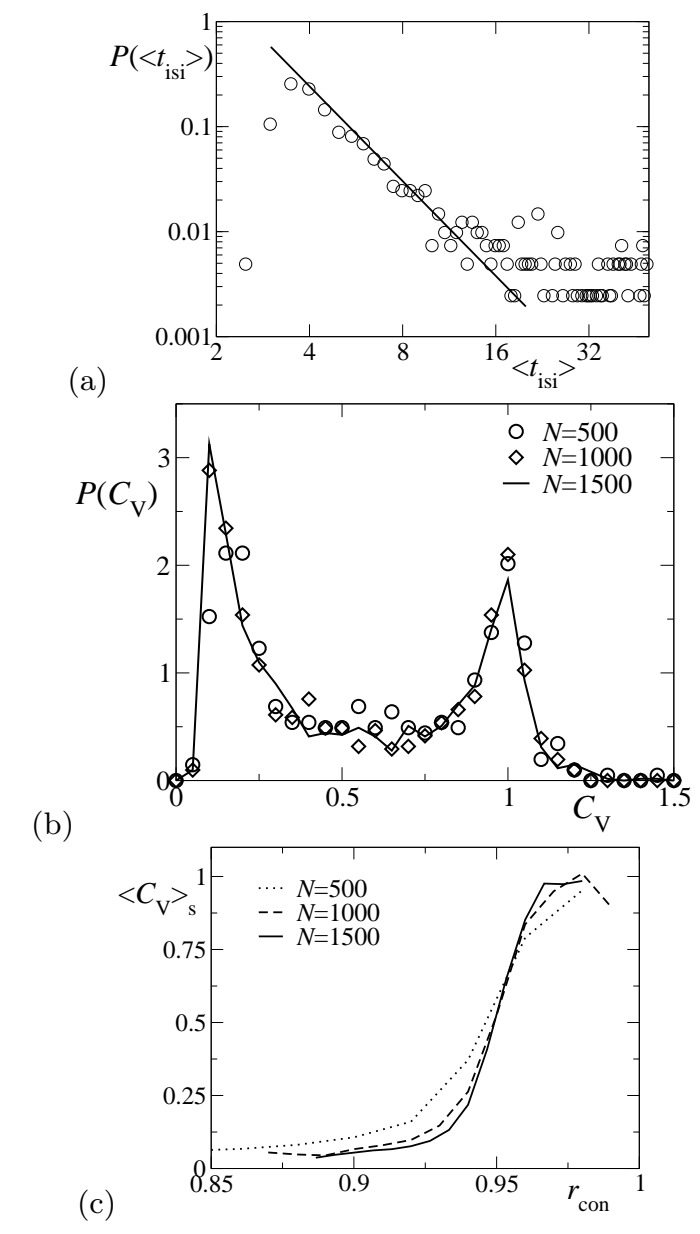

FIG. 4: (a) The stationary distribution of the "background" $\left\langle t_{\text {isi }}\right\rangle$ for the same parameter values as in Fig. 3 (circles). The solid line is a fit with a power-law with exponent 3. (b) The stationary distribution of the $C_{\mathrm{V}}$ of single neurons belonging to the "background" for different system sizes $\left(N^{\prime} / N=0.1\right)$. (c) The average $\left\langle C_{\mathrm{V}}\right\rangle_{\mathrm{s}}$ as a function of the connectivity $r_{\text {con }}$ for different system sizes $\left(N^{\prime} / N=0.1\right)$.

\section{CONCLUSIONS AND PERSPECTIVES}

We have studied a diluted inhibitory network of pulsecoupled neurons that is dynamically stable in the whole parameter range considered. The dynamical stability, determined by a negative Lyapunov exponent, ensures the existence of asymptotic periodic attractors. By varying the coupling strength $G_{0}$, a desynchronization transition is found: for $G_{0} \gtrsim 1.2$ the dynamics is governed by chaotic-like transients whose duration grows exponentially with the system size. The transients have good stationarity properties and they are characterized by irregular firing sequences with an exponentially tailed ISI distribution. Such a behavior is usually attributed to dynamically unstable chaotic networks, e.g., with balanced excitatory and inhibitory activity 11. With the help of mean field arguments, the basic mechanism of the 
desynchronization can be identified: the dropout of input due to a pruned link which leads to irregular changes of the neurons position in the firing sequence. Thus we expect the effect to emerge in a wider class of models (e.g. inhibitory networks with small fractions of excitatory links). Preliminary simulations have further shown the effect to persist in the presence of delayed interaction.

We want to point out that only under the peculiar conditions of stable chaos (SC) it is possible to reconcile linear stability with an unpredictable, chaotic evolution: as soon as the system size is sufficiently large, the periodic attractor is practically unreachable and the erratic transient dynamics is the only accessible information from the system. These findings could be of some relevance in connection with the recent experimental results of Mazor and Laurent [], who pointed out that odor discrimination is better performed by projection neurons in the locust antennal lobe during transient dynamical phases preceeding stable states.

In biological systems, the effect of noise has to be taken into account. In the present case this could manifest itself as external background noise or as internal fluctuations such as synaptic failures. For small enough noise amplitudes, the linear stability of the considered system ensures robustness of the presented behavior. We have shown this by synchronizing a subset of the neurons, where the irregular activity of the other neurons can be seen as weak background noise. In the case of larger noise, one has to consider two cases. In the desynchronized phase $\left(G_{0} \gtrsim 1.2\right)$ the noise amplifies the already present irregularities which renders the convergence towards a periodic attractor impossible. For what concerns the locked phase $\left(G_{0} \lesssim 1.2\right)$, we found that finite perturbations switch the dynamics between the different periodic attractors. However, a detailed investigation of noise effects is still in progress.

The observed mechanisms provided by SC should be present in a dynamical model of a system which is expected to reliably perform universal computations. Indeed, thanks to its asympotic stability, the SC regime is found to be naturally stable with respect to external perturbations. On the other hand, the long and stationary transient ensures that the system can explore an extremely rich variety of configurations.

This gives rise to the challenging question, whether it is possible to address the various periodic states in a reproducible way by properly chosen inputs. In this spirit a study of the properties of the considered dynamics including external drives (information input) is of high interest. As a first test, we have demonstrated that it is possible to lock by external periodic signals a relatively small subset of neurons onto a periodic attractor while the rest (background) of the network performs an irregular activity. Interestingly, the background decomposes into two classes with respect to the $C_{\mathrm{V}}$ of the single neuron ISI distribution, where one class is characterized by a $C_{\mathrm{V}}$ close to unity. All of these remarks indicate that the locked as well as the desynchronized phase of our model may contain ingredients of direct interest for applications to neurosciences.

Finally, we wish to emphasize that from a conceptual point of view, it is remarkable that SC has been found in a continuous-time autonomous dynamical system such as model (11). After its discovery in space- and time-discrete models (coupled map lattices) [8], the only known case of a continous-time dynamical model exhibiting SC is a chain of periodically forced Duffing oscillators [1]. In fact, an autonomous dynamical system depending on a continuous time variable is expected to be marginally stable, i.e., $\Lambda=0$. Such a statement can be made rigorous as soon as the interactions which determine the dynamics of such a system are assumed to be smooth, differentiable functions of its variables. This is not the case of our model, which is certainly continuous in time but incorporates strong nonlinear, i.e., non-smooth, properties due to the threshold mechanism ruling firing events. It is this constituent property, typical of neural dynamics, which essentially turns the continous time dynamics to a map-like behavior, thus rendering possible the emergence of SC.
[1] R. Bonaccini, A. Politi, Chaotic-like behaviour in chains of stable nonlinear oscillators Physica D 103 (1997) 362368.

[2] N. Brunel, V. Hakim, Fast global oscillations in networks of integrate-and-fire neurons with low firing rates, Neural Computation 11 (1999) 1621-1671.

[3] N. Brunel, X.-J. Wang, Effects of neuromodulation in a cortical network model of object working memory dominated by recurrent inhibition, J. Comput. Neurosci. 11 (2001) 63-85.

[4] A. Destexhe, M. Rudolph, J.-M. Fellous, T. J. Sejnowski, Fluctuating synaptic conductances recreate in vivo-like activity in neocortical neurons, Neuroscience 107 (2001) $13-24$.

[5] W. Gerstner, W. M. Kistler, Spiking neuron models, Cambridge Univ. Press, Cambridge, 2002.
[6] D. Z. Jin, Fast convergence of spike sequences to periodic patterns in recurrent networks, Phys. Rev. Lett. 89 (2002) 208102.

[7] O. Mazor and G. Laurent, Transient dynamics versus fixed points in odor representations by locust antennal lobe projection neurons, Neuron 48 (2005) 661-673.

[8] A. Politi, R. Livi, G.-L. Oppo, R. Kapral, Unpredictable behaviour of stable systems, Europhys. Lett. 22 (1993) 571-576.

[9] W. R. Softky, C. Koch, The highly irregular firing of cortical cells is inconsistent with temporal integration of random EPSPs, J. Neurosci. 13 (1993) 334-350.

[10] H. C. Tuckwell, Introduction to theoretical neurobiology, Cambridge University Press, New York, 1988.

[11] C. van Vreeswijk, H. Sompolinsky, Chaos in Neuronal Networks with Balanced Excitatory and Inhibitory Ac- 
tivity, Science 274 (1996) 1724-1726.

[12] R. Zillmer, R. Livi, A. Politi, A. Torcini, Desynchronization in diluted neural networks, cond-mat/0603154

[13] A. Zumdieck, M. Timme,T. Geisel, F. Wolf, Long chaotic transients in complex networks, Phys. Rev. Lett. 93 (2004) 244103. 